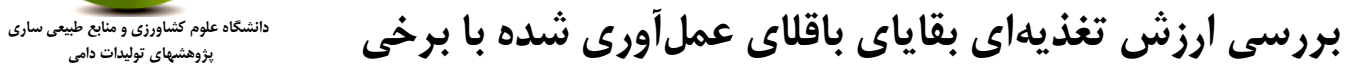

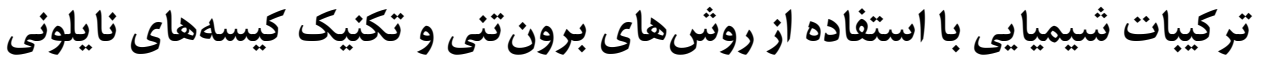

\author{
آذين علايى'، فرزاد قنبرى ؟، جواد بيات كوهسارّ و فريبا فريورّ

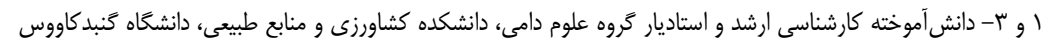

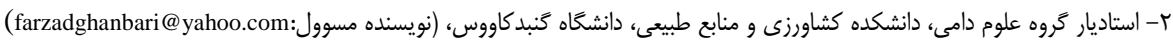 \\ تاريخ دريافت: \\ صفحه: 19 تا
}

جكيده

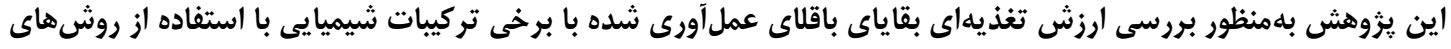

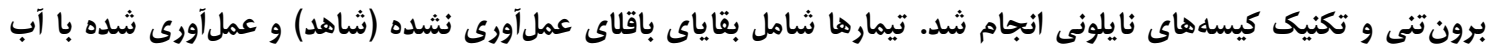

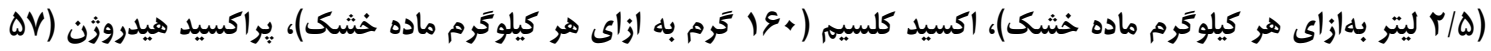

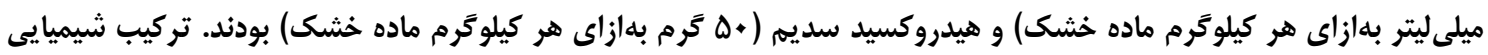

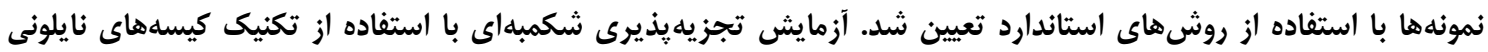

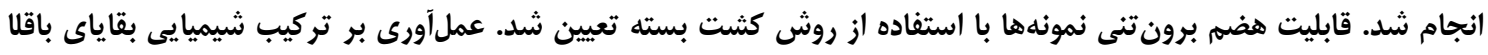

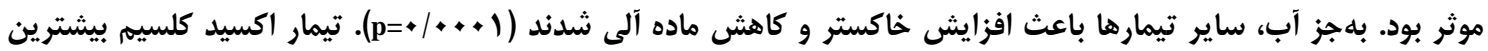

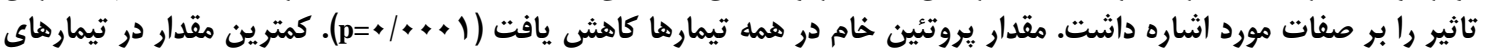

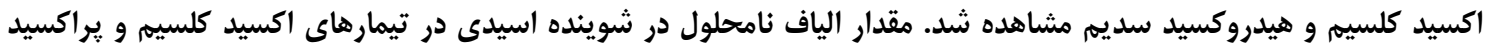

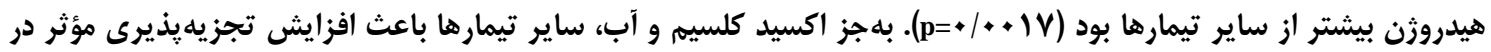

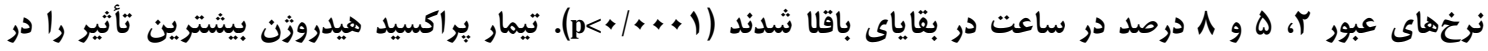

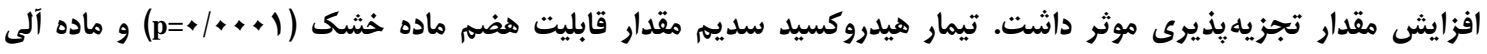

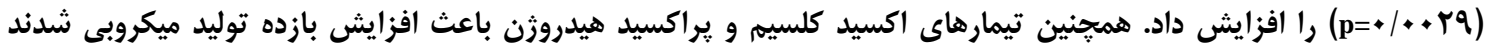

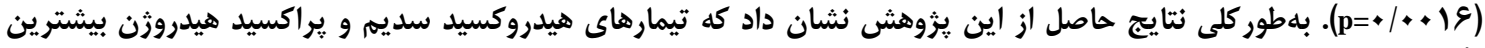
تأثير را در بهبود ارزش بهرك تغذيهاى بقاياى باقلا داشتند.

وازههاى كليدى: عمل آورى، تركيبات شيميايى، قابليت هضم، تجزيه يذيرى شكمبهاى، بقاياى باقلا

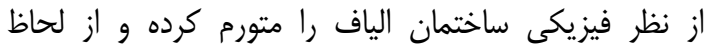

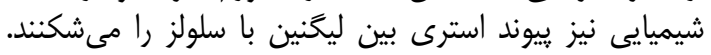

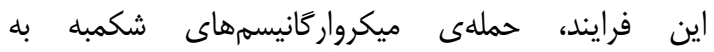

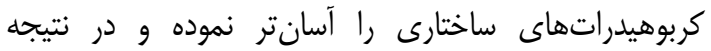

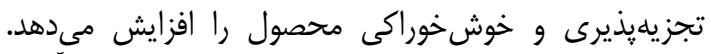

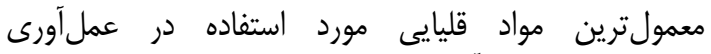

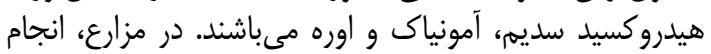

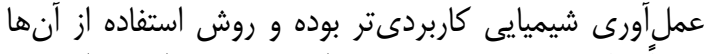

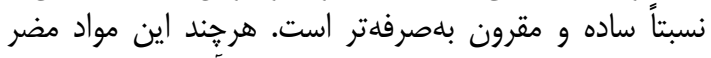

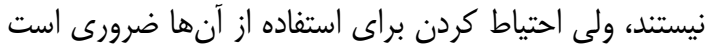

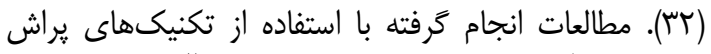

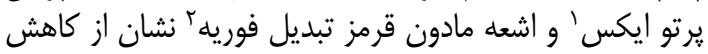

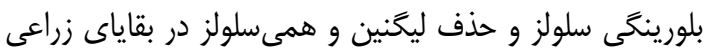

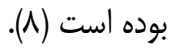

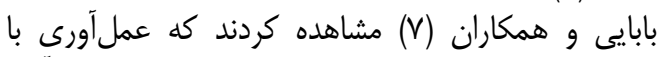

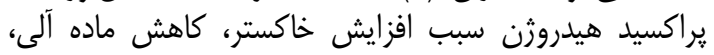

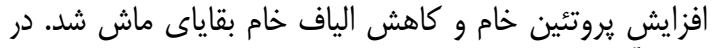

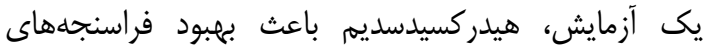

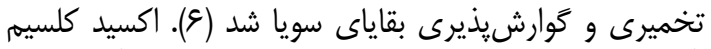
(آهك)، بهدليل مزاياى زيادى كه دارد، براى عمل آورى لئى مواد

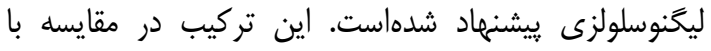

مقدمه امروزه با توجه به كمبود منابع خوراكى اصلى و وزئه هزينه

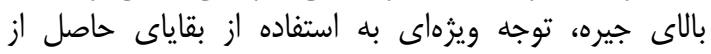

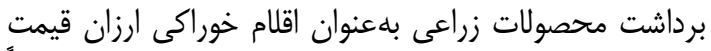

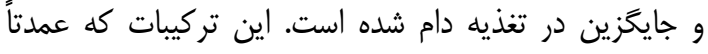

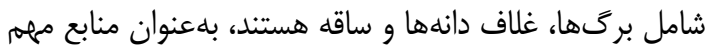

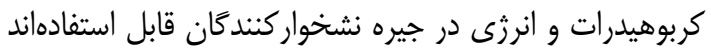

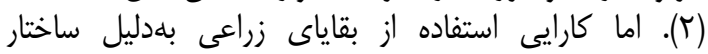

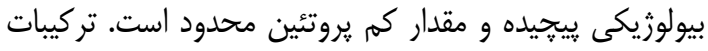

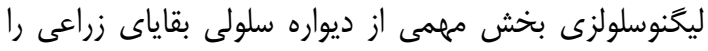

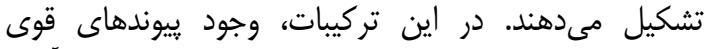

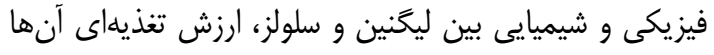

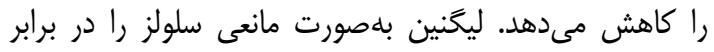

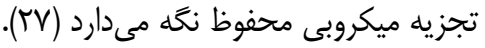

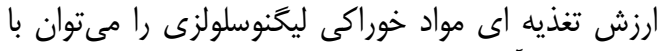

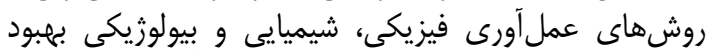

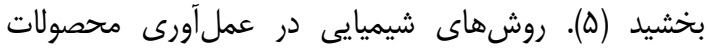

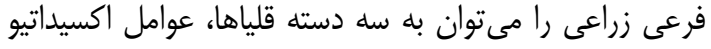

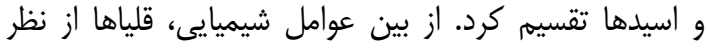

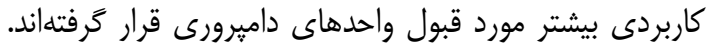

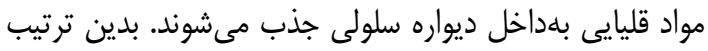




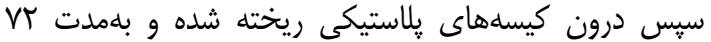

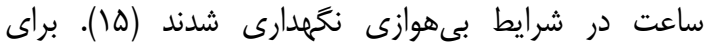

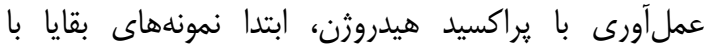

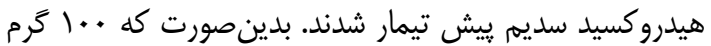

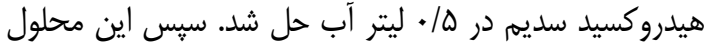

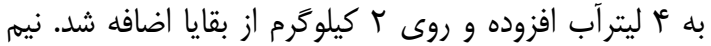

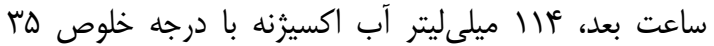

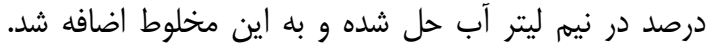

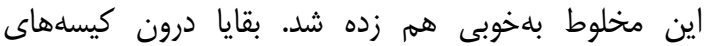

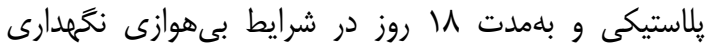

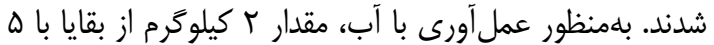

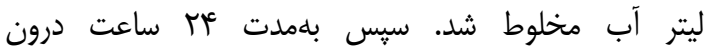

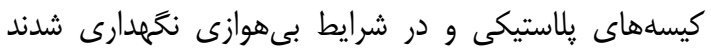

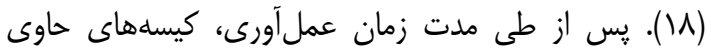

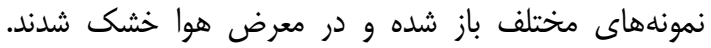

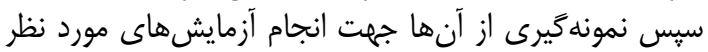

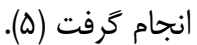

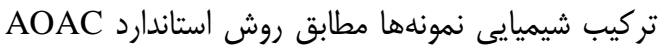

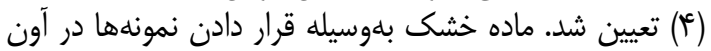

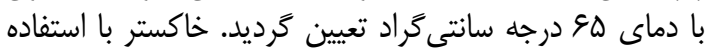

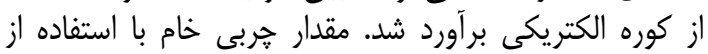

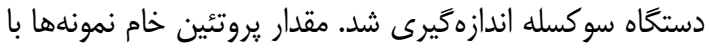

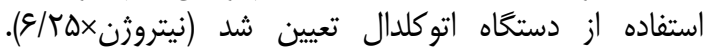

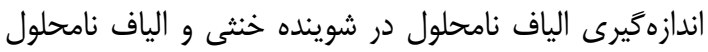

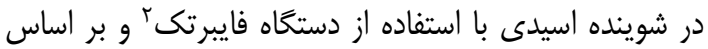
روش ون سوست (باء) انجام شد.

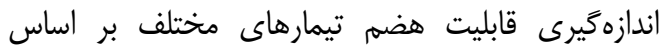

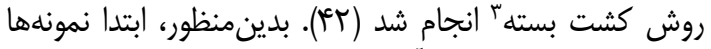

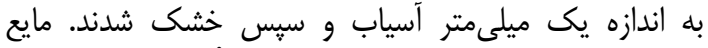

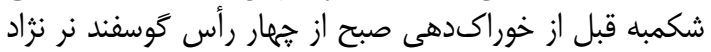

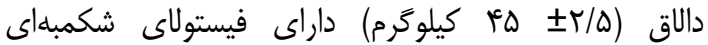

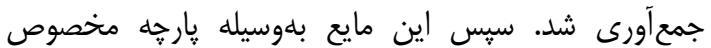

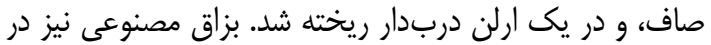

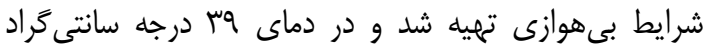

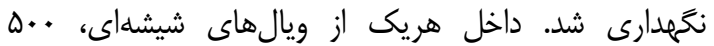

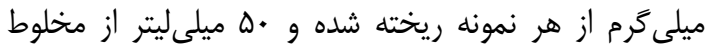

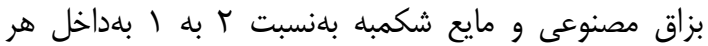

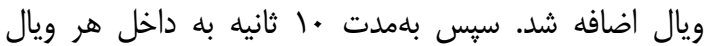

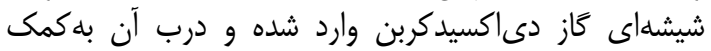

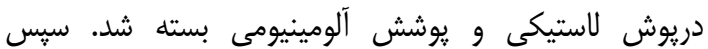

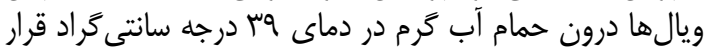

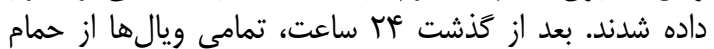

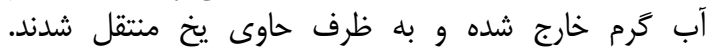

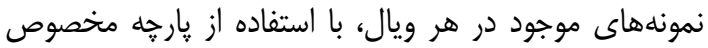

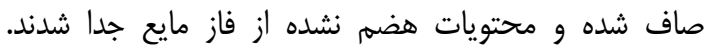

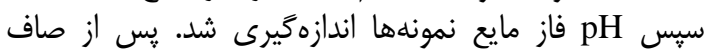

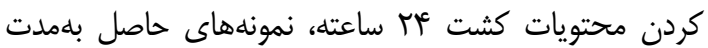

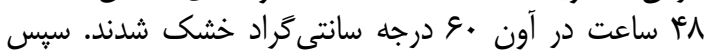

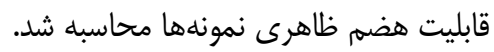

هيدروكسيد سديم ارزانتر و سالمتر بوده و ماده شيميايى ضعيفترى نسبت به آن است. بنابراين استفاده از آن آن نياز به به إنه

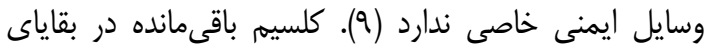

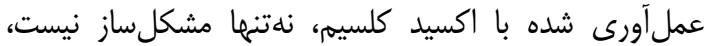

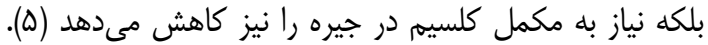

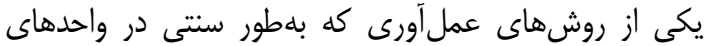

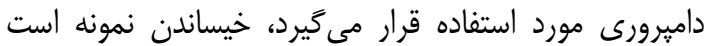

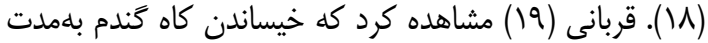

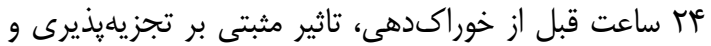

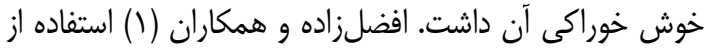

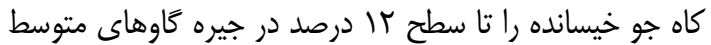
توليد بيشنهاد كردند.

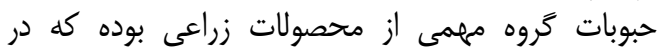

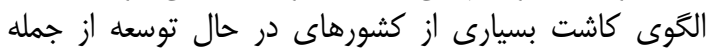

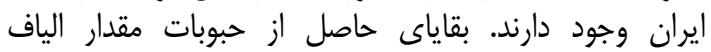

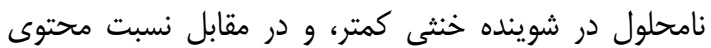

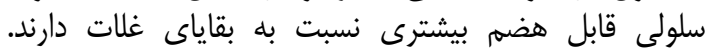

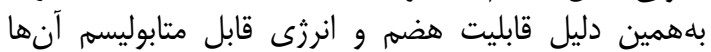
بيشتر از بقاياى غلات مى بـاشد (هّ)

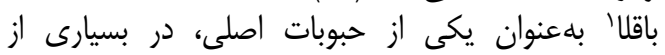

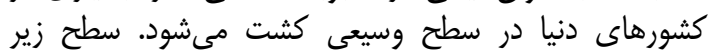

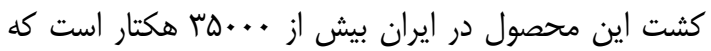

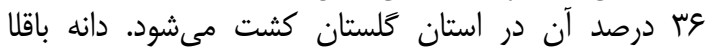
بلهنوان محصول اصلى براى مصارف انسانى مورد استفاده

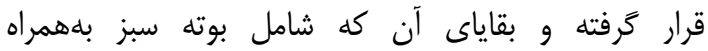

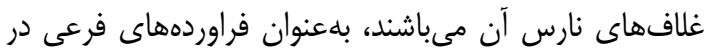

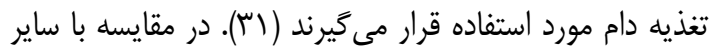

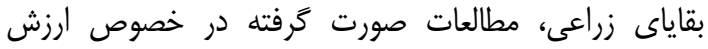
تغذيهاى بقاياى باقلا محدود است.

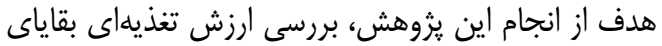

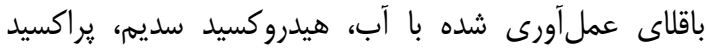

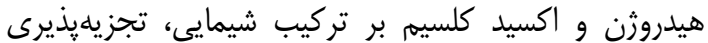
شكمبهاى و قابليت هضم برون تنى بقاياى باقلا بود.

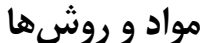

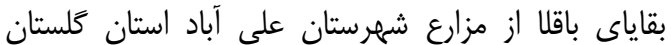

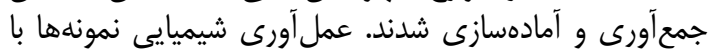

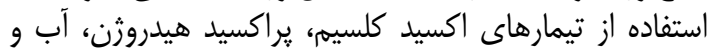

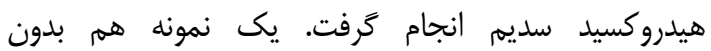

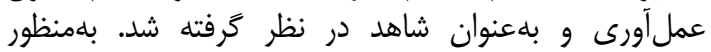

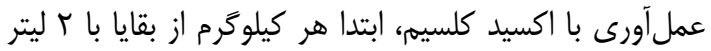

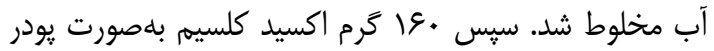

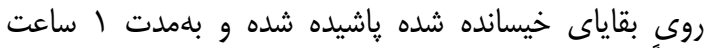

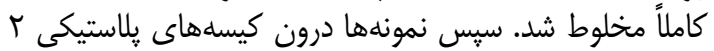

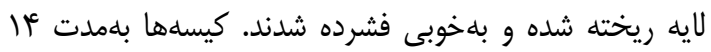

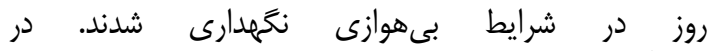

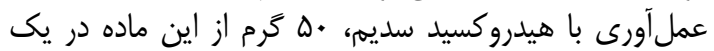

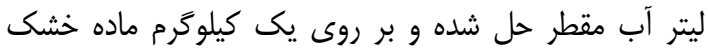

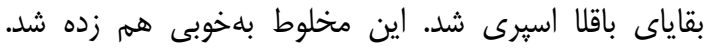


در اين رابطه ERD: تجزيهيذيرى موثر و k: نرخ عبط مبور

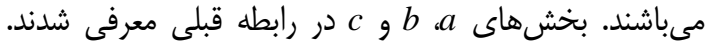
تجزيه واريانس دادههاى مربوط باله به تركيب شيميايى و و قابليت

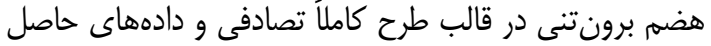

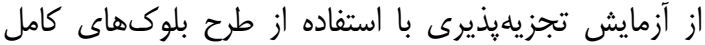

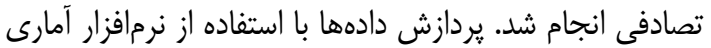
SAS ميانگينها از آزمون حداقل تفاوت معنىدار' استفاده شد.

\section{نتايج و بحث تركيب شيميايى \\ تأثير تيمارهاى اكسيد كلسيه، : يراكسيد هيدروزن،}

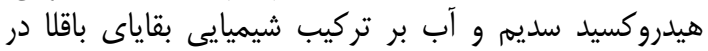

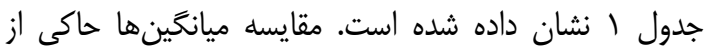

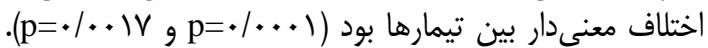

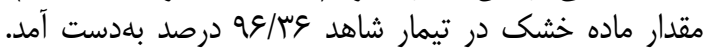

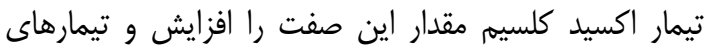

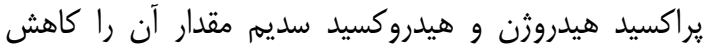
دادند (بهترتيب

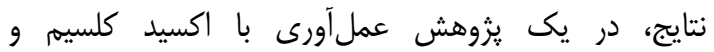

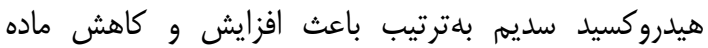

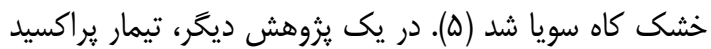

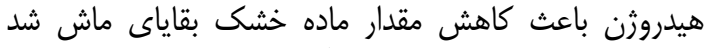

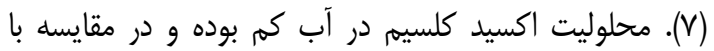
ساير تركيبات شيميايى مانند هيدروكسيد سديم آيم نياز به به مقدار

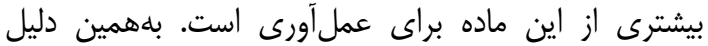

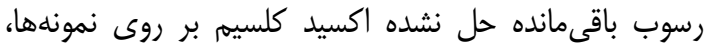

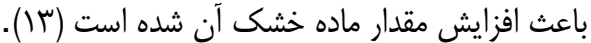

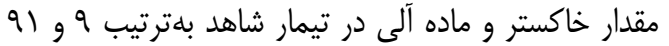

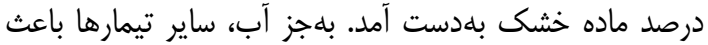
افزايش مقدار خاكستر و كاهش ماده ماده آلى شدند. تئد تيمار اكسيد

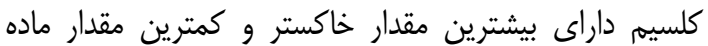

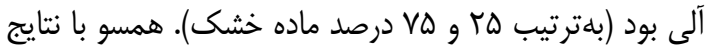

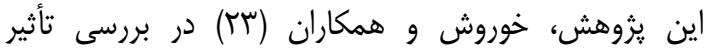

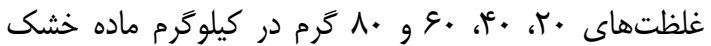

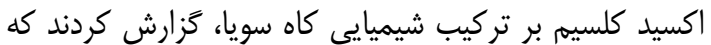

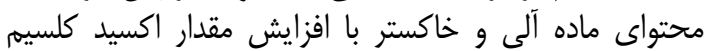

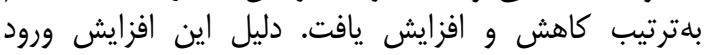

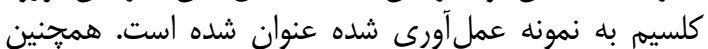

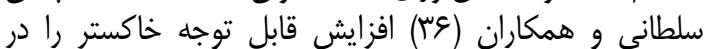

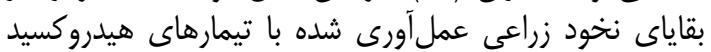

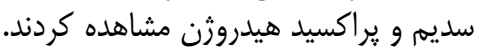

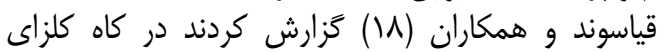
عمل آورى شده با هيدروكسيد سديه، دروصد ماده آلى كانى كاهش و

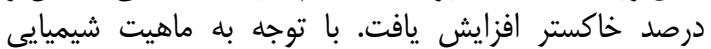

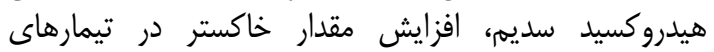

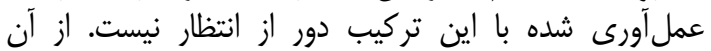

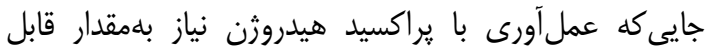
توجهى هيدروكسيد سديم بلمنظور حفظ pH در محدودمى يليد
مقدار نيتروزن أمونياكى نمونهها با استفاده از روش

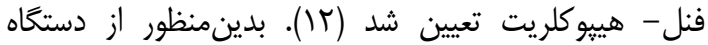

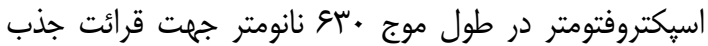

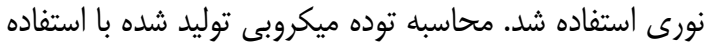

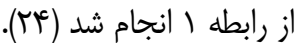

رابطه (1)

$\mathrm{MB}=\mathrm{GP} \times(\mathrm{PF}-\mathrm{T} / \mathrm{T})$

در اين رابطه، MB: توليد توده ميكروبى (ميلى گرم)،

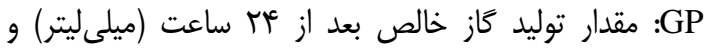

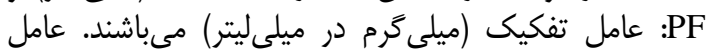

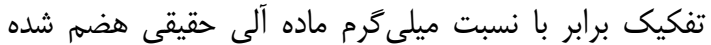

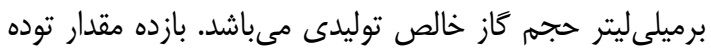

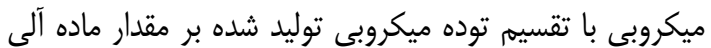

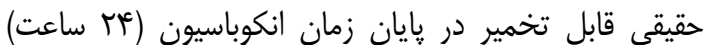
محاسبه شد.

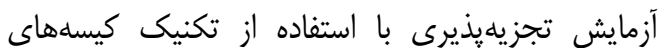

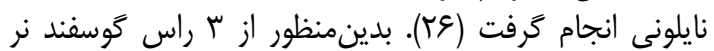

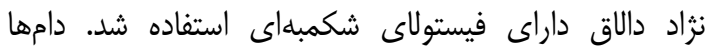

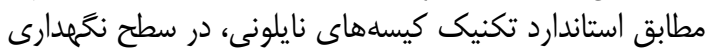

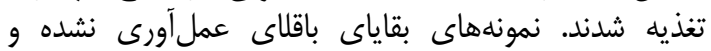

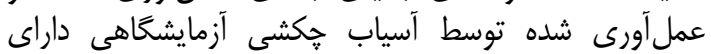

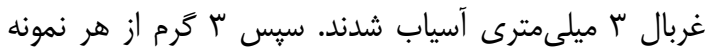

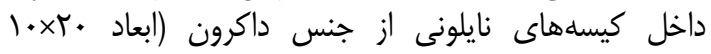

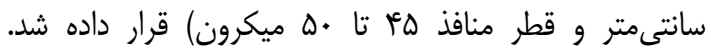

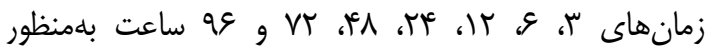

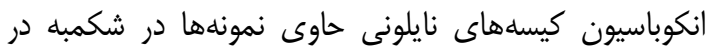

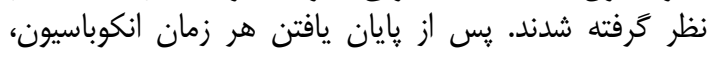

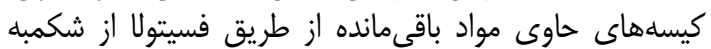

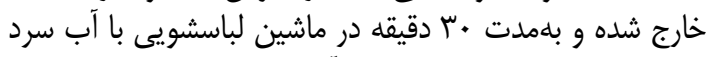

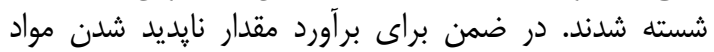
خوراكى در زمان صفر يا مقدار مواد محلول درا در آب، كيسههاي

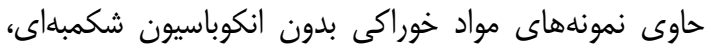

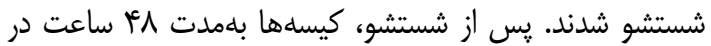
آون 9Q درجه خشك شداند. در نهايت با با تعيين مقدار نمونه

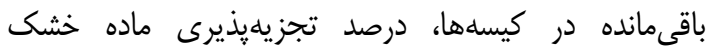

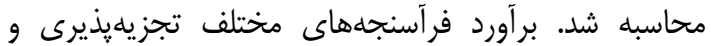

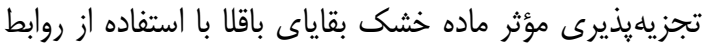

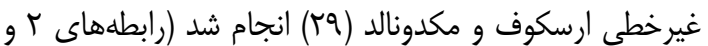

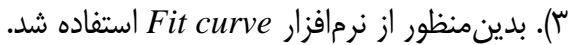

$\mathrm{P}=a+b\left(1-\mathrm{e}^{-c t}\right)$ رابطه (T)

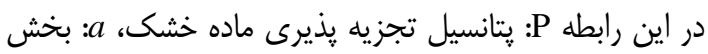

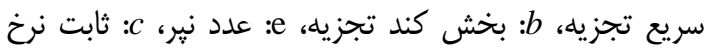
تجزيه و t: مدت زمات زمان قرار دادن نمونه ديه در شكمبه

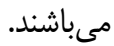
رابطه (")

$E R D=a+\left[\frac{b \times c}{c \times k}\right]$ 
در اين يزوهش مقدار الياف نامحلول در شوينده خنثى در

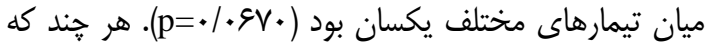

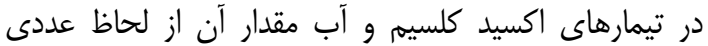

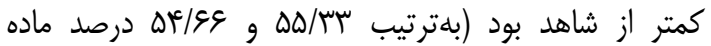

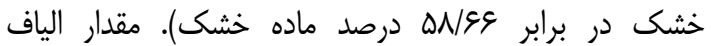
نامحلول در شوينده اسيدى در تيمارهاى اكسيد كليد بلسيم و

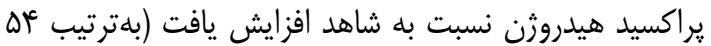

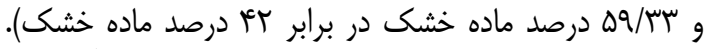

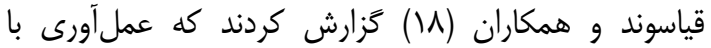

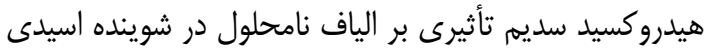

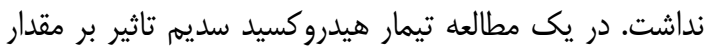

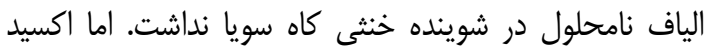

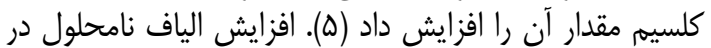

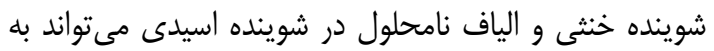

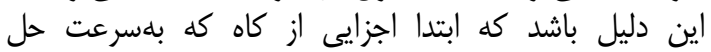

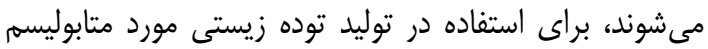

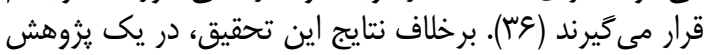

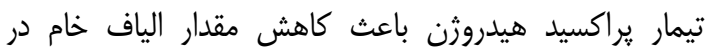
بقاياى ماش شد كه دليل آن حذف بخش كائ عمده ليدار ليخنين و

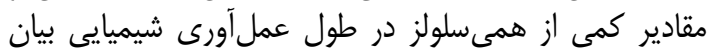

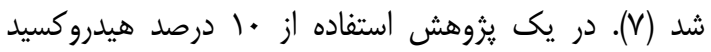

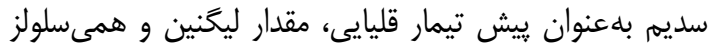

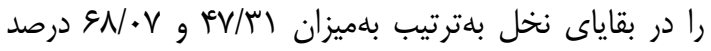

$$
\text { كاهش داد (^). }
$$

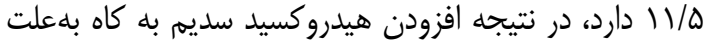
اثرات باقىمانده اين ماده، منجر به افز إيش درديد إند خاكستر خام

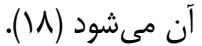

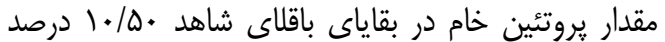

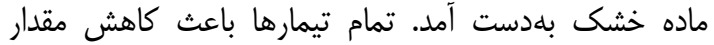

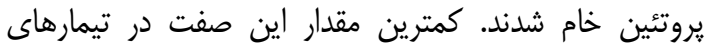

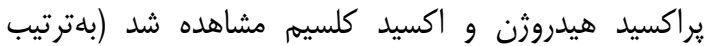

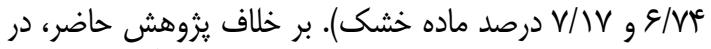

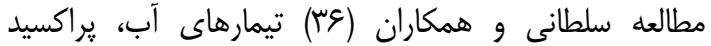

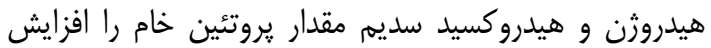

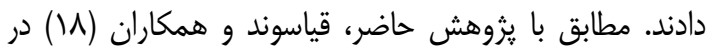

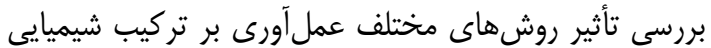

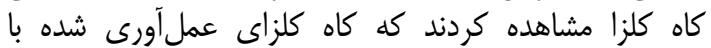

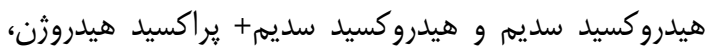
درصد يروتئين خام كمترى نسبت به به شاهد داشيد داشتند. افزودن

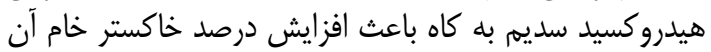

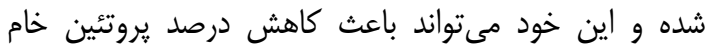

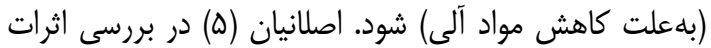

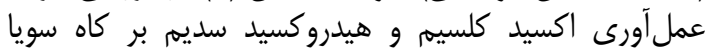
كزارش كرد كه عمل آورى با اين تيمارها باعث كارئ كاهش مقدار

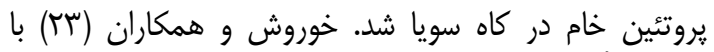

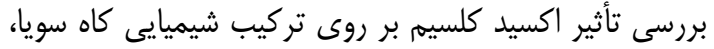

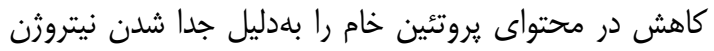
از اجزاى تشكيلدهنده كاه بيان داشتند.

جدول ا- تأثير اكسيد كلسيه، يراكسيد هيدروثن، هيدروكسيد سديم و آب بر تركيب شيميايى بقاياى باقلا (درصد ماده خشك)

Table 1. Effect of calcium oxide, hydrogen peroxide, sodium hydroxide and water on chemical composition of vicia

\begin{tabular}{|c|c|c|c|c|c|c|}
\hline در شوينده اسيدى & شوينده خنثى & يروتئين خام & ماده آلى & خاكستر & ماده خشك & تيمارها \\
\hline$F T / . .^{b}$ & $\Delta \mathrm{N} / q^{\mathrm{ab}}$ & $1 \cdot 10 \cdot \cdot^{a}$ & $91 / \cdots^{a}$ & $9 / .^{d}$ & aq/\% & شاهد \\
\hline$\Delta F / . a^{a}$ & $\Delta \omega / \mu \mathrm{rb}$ & $\mathrm{V} / \mathrm{v} \cdot \mathrm{cd}$ & $V Q / . . \mathrm{d}^{\mathrm{d}}$ & $r \Delta / .{ }^{a}$ & $q \vee / / V^{a}$ & اكسيد كلسيم \\
\hline$\Delta q / m \mu^{a}$ & $9.1 . .^{a}$ & $s / V^{e d}$ & $V N / q)^{c}$ & $r M / \wedge^{b}$ & $q \Delta / r \gamma^{c}$ & يراكسيد هيدروثن \\
\hline$F+/ . . b$ & $\Delta f / \varepsilon, b$ & $q / r \Lambda^{b}$ & $9 . / 9)^{a}$ & $q / \wedge^{d}$ & $q / g \varphi^{a b}$ & "آب \\
\hline$|c| . .^{b}$ & $9 \cdot 1 . .^{a}$ & $V / M^{c}$ & $\Lambda \bowtie / . . \mathrm{b}$ & $\mid \omega / . .^{c}$ & $q \Delta / q^{c}$ & هيدروكسيد سديم \\
\hline T/RT. & $1 / 94$. & $\cdot / \pi E^{2}$ &.$/ 94$. &.$/ 94$. & $\cdot / \pi \cdot$ & اشتباه معيار ميانگين \\
\hline $.1 \cdot .1 \mathrm{~V}$ & $.1 .9 \mathrm{~V}$. & $.1 \ldots 1$ & $\cdot|\cdots|$ &.$|\cdots|$ &.$|\cdots|$ & اختلاف معنىدارى \\
\hline
\end{tabular}

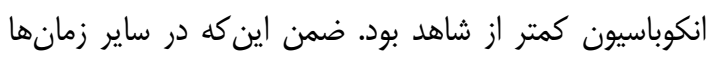

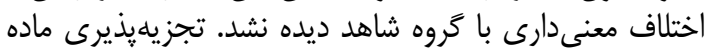

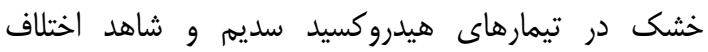
معنى دارى با هم نداشت.

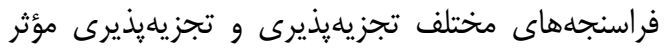

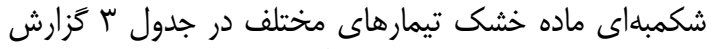

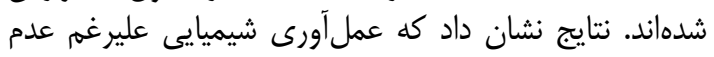

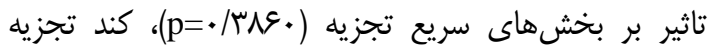

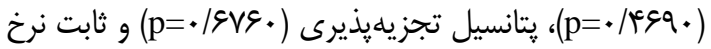

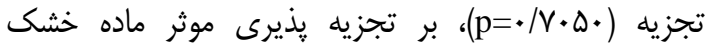

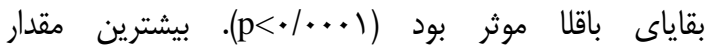

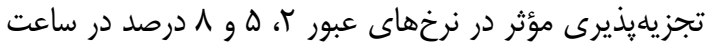

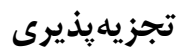

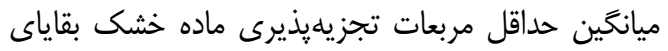

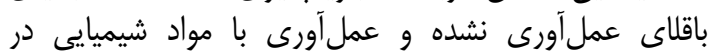

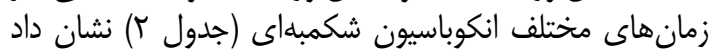

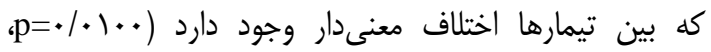

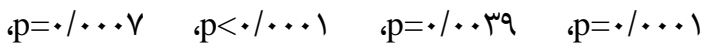
raf

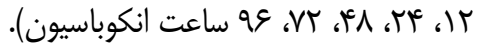

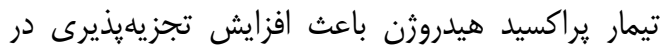

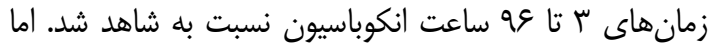

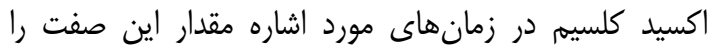

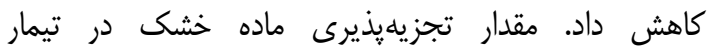
عملآورى شده با آب در زمانهاى 
الكلى بهداخل ديواره سلولى و شكستن ييوندهاى استرى ميان

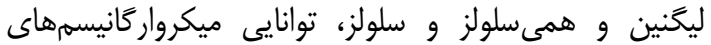

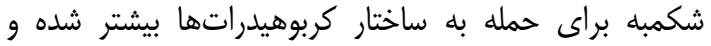

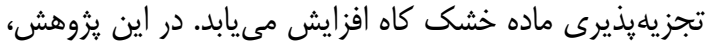

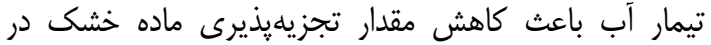

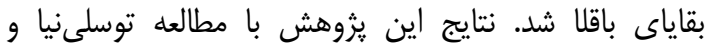

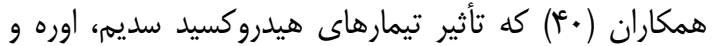

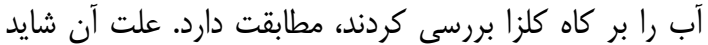

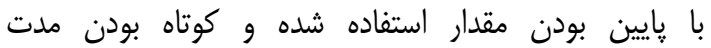

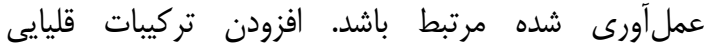

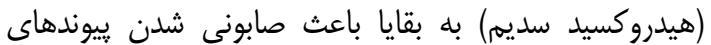

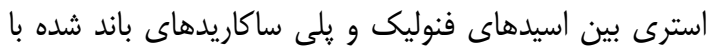

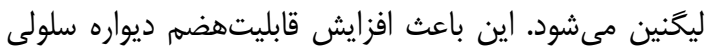

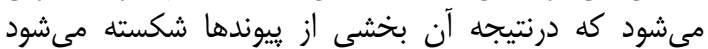

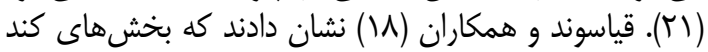

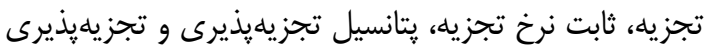

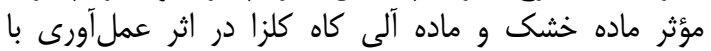

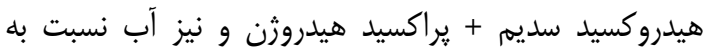

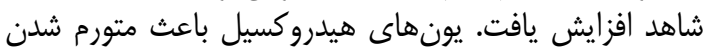

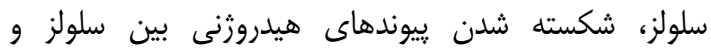
همىسلولز و هيدروليز ييوندهاى استرى يلى يلى ساكاريدهاى

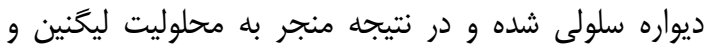

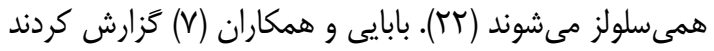

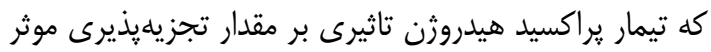

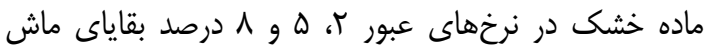

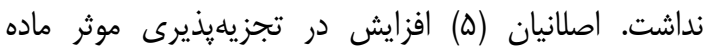

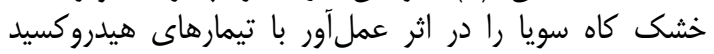

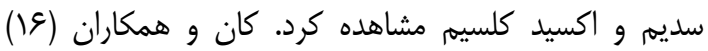

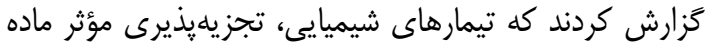

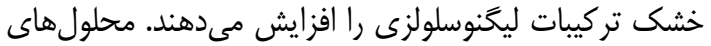

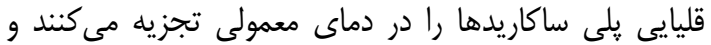

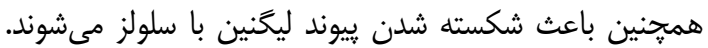

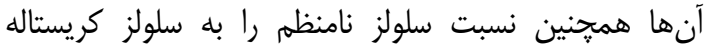

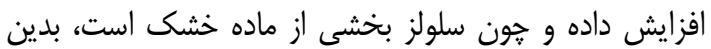

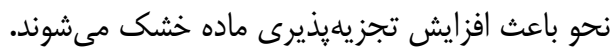

در تيمار يراكسيد هيدروثن، و كمترين مقدار آن در تيمار اكسيد كلسيم مشاهده شد.

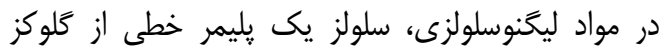

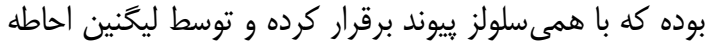

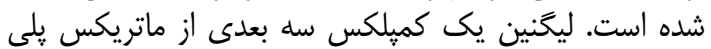

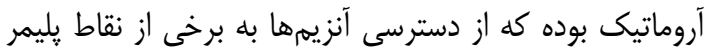

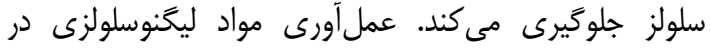

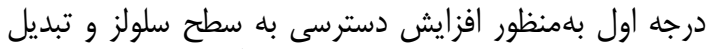

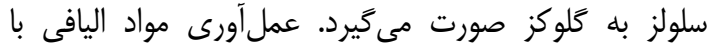

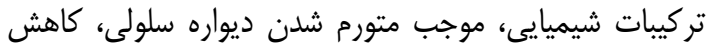

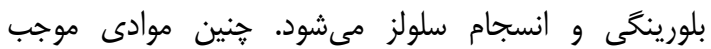

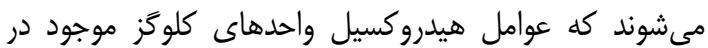

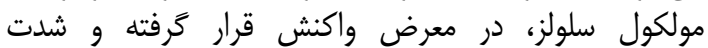

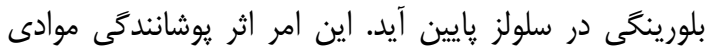

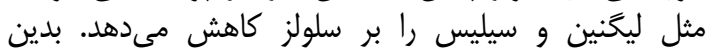

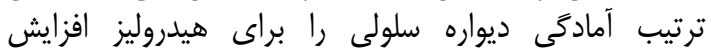

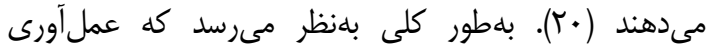

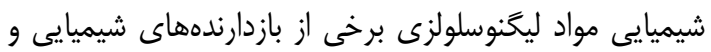

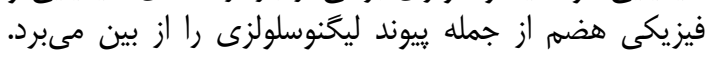

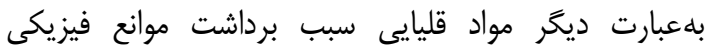

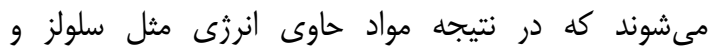

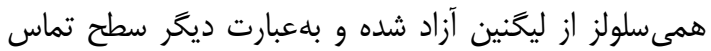

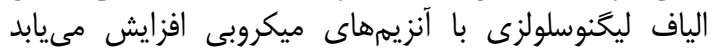

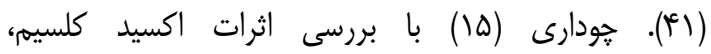

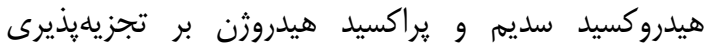

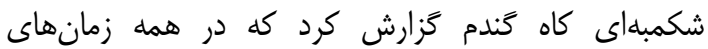

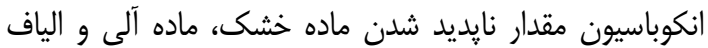

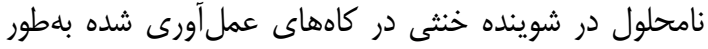

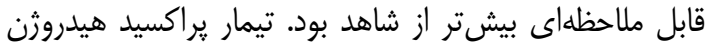

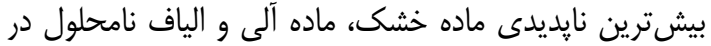

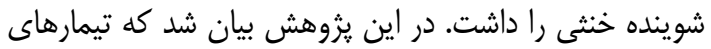

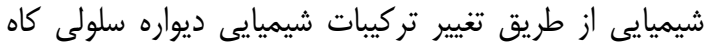

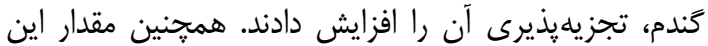

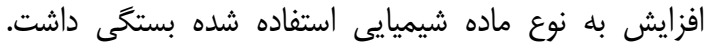

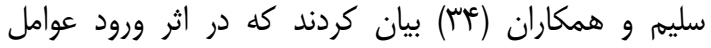


جدول r- تأثير اكسيد كلسيم، يراكسيد هيدروثن، هيدروكسيد سديم و آب بر روند تجريهيذيرى ماده خشى بقاياى باقلا در زمانهاى مختلف انكوباسيون (درصد)

Table 2. Effect of calcium oxide, hydrogen peroxide, sodium hydroxide and water on dry matter degradability trend of vicia faba residues at different times of incubation (percent)

\begin{tabular}{|c|c|c|c|c|c|c|c|c|}
\hline \multicolumn{8}{|c|}{ زمان انكوباسيون(ساعت) } & \multirow{2}{*}{ تيمارها } \\
\hline 98 & vr & \&^ & Tr & ir & 9 & r & صفر & \\
\hline$r+/ \Lambda e^{c b}$ & $r q / v \Lambda^{a b}$ & $\Psi N / \mathcal{A N}^{\mathrm{b}}$ & 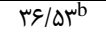 & $r F / V r^{b}$ & $\Gamma \cdot / \widetilde{\mu} \Delta^{b}$ & $r V / l f^{c a b}$ & $r M / r V^{a}$ & شاهد \\
\hline$\Delta \backslash / \mathbb{E}^{\mathrm{a}}$ & $\Delta T / F^{a} I^{\mathrm{a}}$ & $\leftarrow \varepsilon / V T^{a}$ & $f+/ l \in a$ & $\leftarrow \varepsilon / v \cdot a^{a}$ & $r \Delta / \varphi^{e c a}$ & $r \cdot / \Delta \varphi^{a}$ & $r m / r e a$ & يراكسيدهيدروثن \\
\hline $\mathrm{r} N \cdot \wedge^{\mathrm{c}}$ & cl & $r T / 1 q^{c}$ & $r \cdot|\Delta|^{c}$ & $r V / V \cdot{ }^{c b}$ & $r \Delta / \omega \cdot c$ & $r \Psi / V \Lambda^{c b}$ & r)/qua & آب \\
\hline$r \varepsilon / V^{\mathrm{ab}}$ & $c \cdot 1 \cdot q^{a b}$ & $r q / v \varphi^{c b}$ & $r \vee / \wedge q^{b}$ & $r F / / V^{b}$ & $r \cdot 1 q^{b}$ & $r \Lambda / T \Delta^{a b}$ & $r r / \varepsilon \Lambda^{a}$ & هيدرو كسيدسديم \\
\hline.$/ \cdot \Delta F$ & . I. FAT & $\cdot 1 \cdots v$ & $<\cdot|\cdots \cdot|$ & . . . rq & 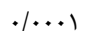 &.$/ \cdot 1$. & $\cdot / \uparrow \wedge .$. & اختلاف معنىدارى \\
\hline
\end{tabular}

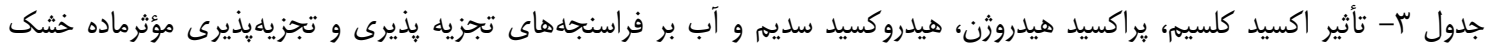
بقاياى باقلا

Table 3. Effect of calcium oxide, hydrogen peroxide, sodium hydroxide and water on degradability parameters and

\begin{tabular}{|c|c|c|c|c|c|c|c|}
\hline \multicolumn{3}{|c|}{ در نجزيهذيذيرى مؤثر (درصد) } & \multirow{2}{*}{ (درصد درساعت) } & \multirow{2}{*}{ تجزيذهيذيرى } & \multirow{2}{*}{ تجزيه (درصد كند } & \multirow{2}{*}{ تجزيه (درصد سريع) } & \multirow[t]{2}{*}{ تيمارها } \\
\hline$\wedge$ & 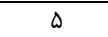 & $r$ & & & & & \\
\hline$\mu / v \cdot b$ & $r \mu / \Delta \& b$ & $r \varepsilon / q \cdot b$ & $4 \cdot 1 \cdot 1$ &.$/ 1$ & $M / T A$ & $r / / v q$ & شاهد \\
\hline$r M / l \cdot d$ & $r r / l \cdot d$ & $r F / g r d$ & $r \Delta / \cdot v$ & $\cdot / \cdot v$ & $I V / \Delta D$ & $|V| \Delta \mid$ & اكسيدكلسيم \\
\hline$r / / v r a$ & ri/fra & ro/s\&a & $0 . / F \mathrm{~V}$ &.$/ T$ & TV/AT & Tr/gr & קراكسيدهيدروثن \\
\hline$r \varepsilon / \wedge \& \mathrm{c}$ & rN/KC & r & FT/AD & .1 .4 & $r \cdot|\Lambda|$ & $r r / \cdot F$ & آب \\
\hline$r r / .9 b$ & $r F / / r b$ & $r N / l \cdot b$ & $\kappa \psi / .$. & .1 .9 & $19 / 41$ & $T E / T \Lambda$ & هيدروكسيدسديم \\
\hline ./vrq & •/VGr & $1 / 19 V$ & S/TVK & 1.45 & $D / \Psi A C$ & r/NF. & شتباه معيار ميانكين \\
\hline$<\cdot / \cdots+1$ & $<\cdot|\cdots \cdot|$ & $<\cdot|\cdots \cdot|$ & $\cdot / V \cdot \Delta \cdot$ & .lavg. &.$/ 499$. & . & اختلاف معنىدارى \\
\hline
\end{tabular}

بلوسيله تركيبات شيميايى حذف يا محدود مى شود. بيان شده

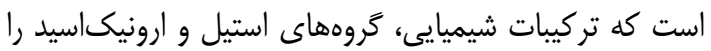

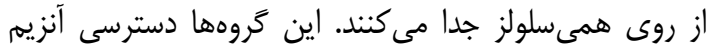

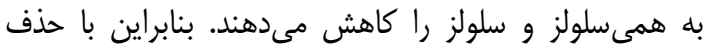

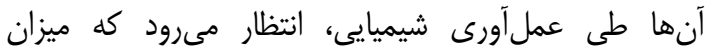

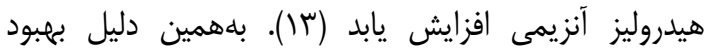

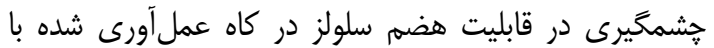

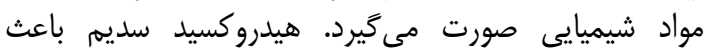

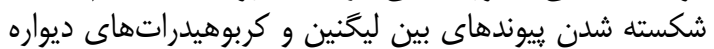

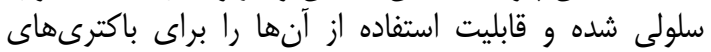

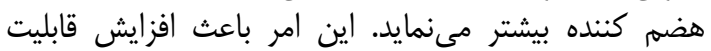

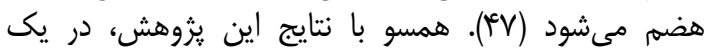

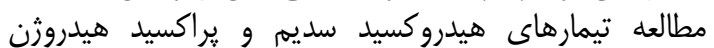

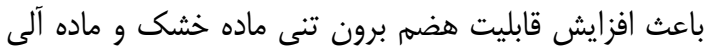

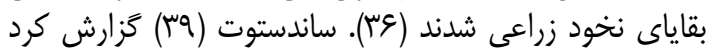

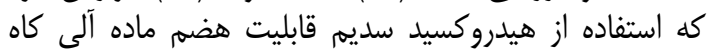

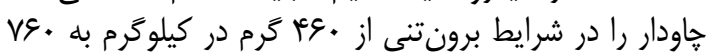

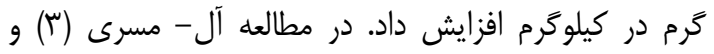

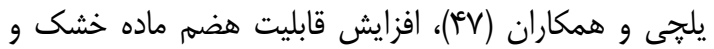

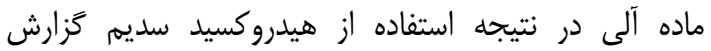

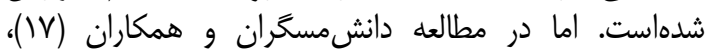
بهبودى در قابليت هضم ماده آلى در اثر افزودن هيدروكسيد
فر اسنجههاى تخميرى

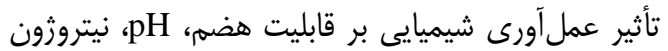

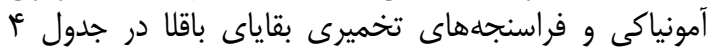

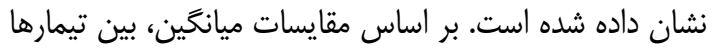

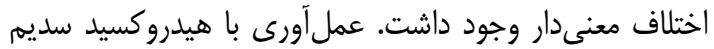

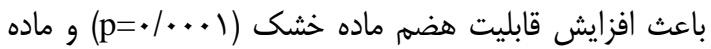

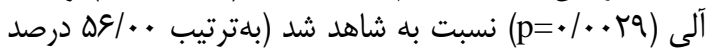

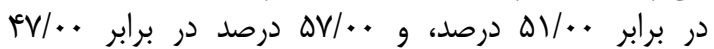

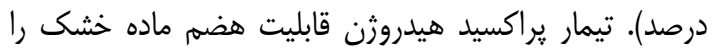

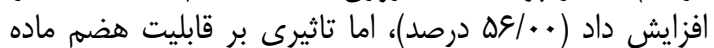

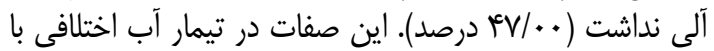

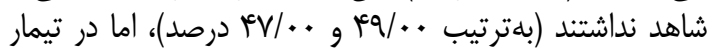

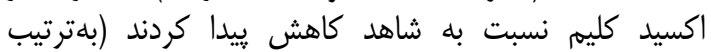

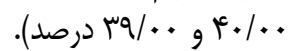

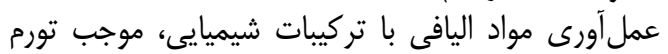

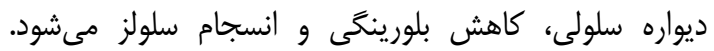

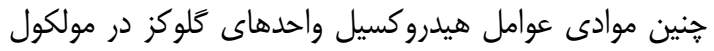

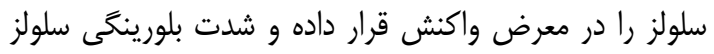

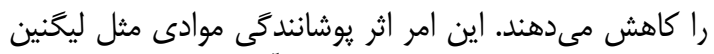

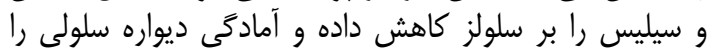

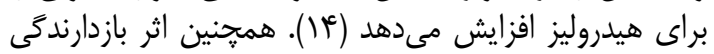

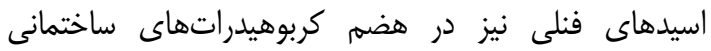


و تشكيل كربنات سديم شده و در نتيجه pH كاه افزايش

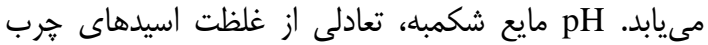

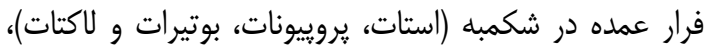

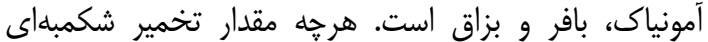

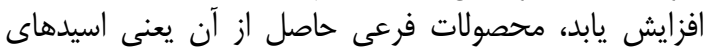

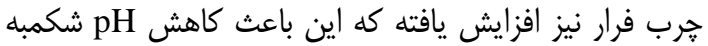

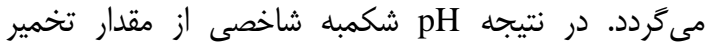

شكمبه است (عثا).

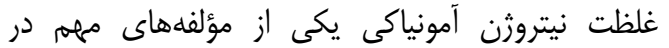

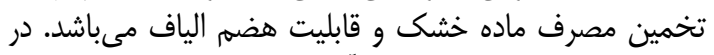
اين يزوهش، غلظت نيتروزن أمونياكى شكمبه دئ در تيمارهاى

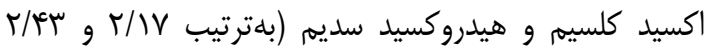

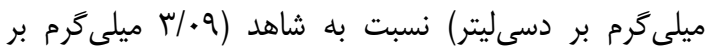

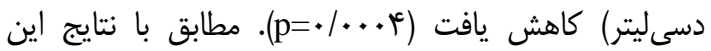

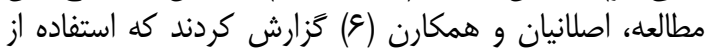

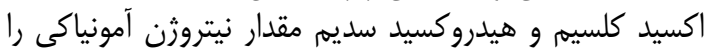
در كاه سويا كاهش داديم هيدروكي

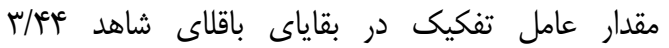

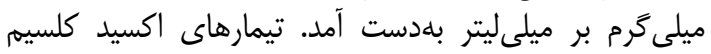

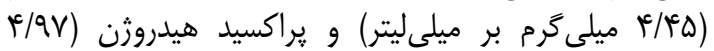
ميلى گرم بر ميلىليتر) باعث افزايش مقدار اين صفت شدند (p=./..14)

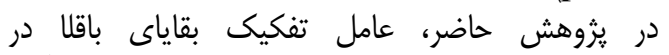

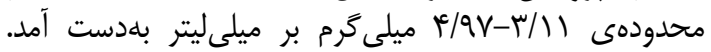

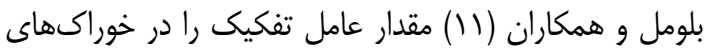

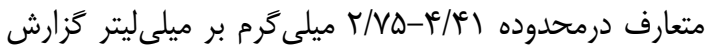

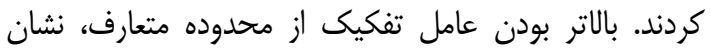

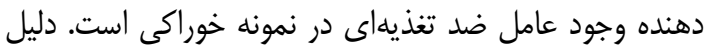

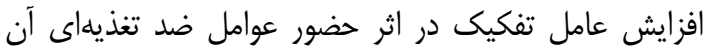

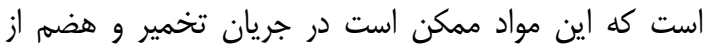

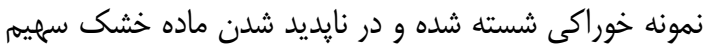

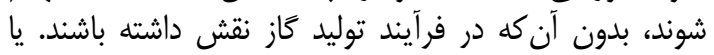

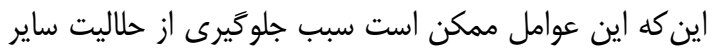

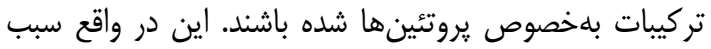

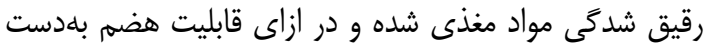

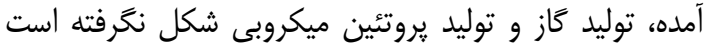

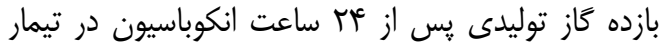

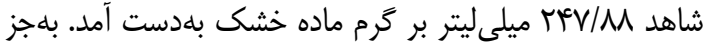

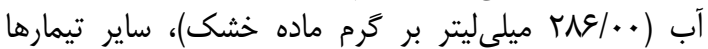

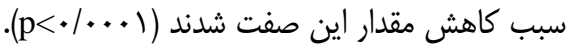

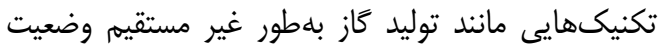

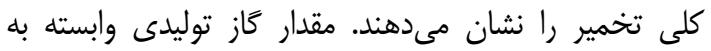

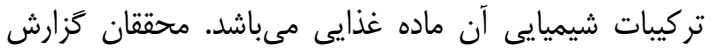

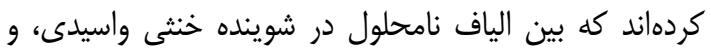

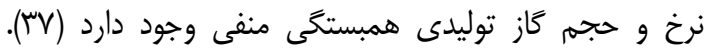

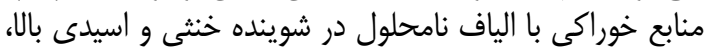

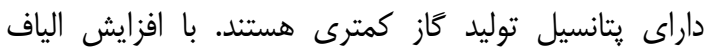

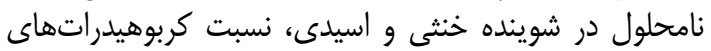

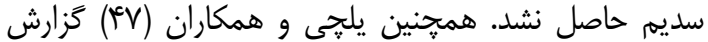

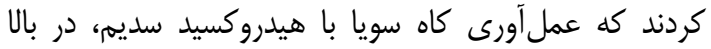

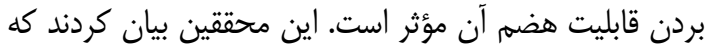

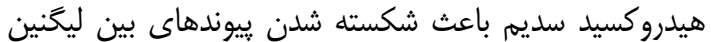

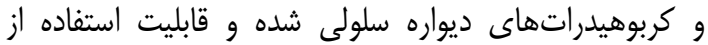

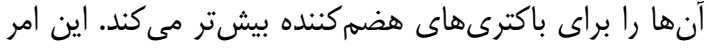

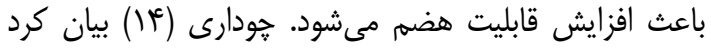

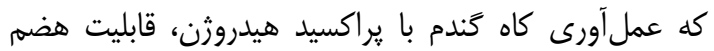

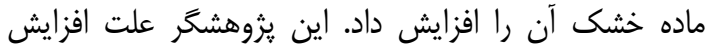

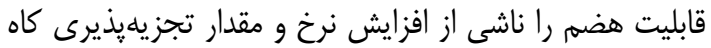

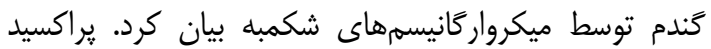

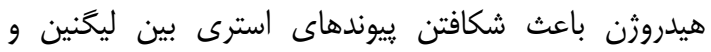

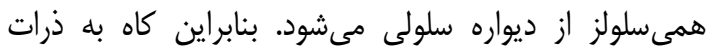

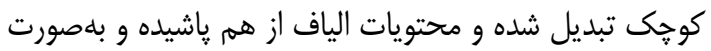

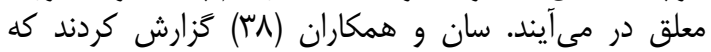

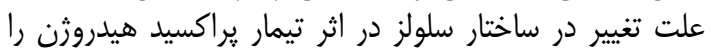

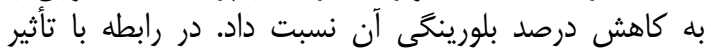

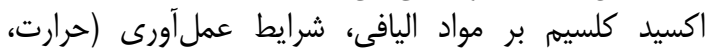

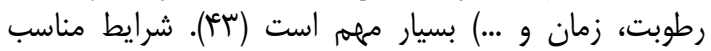

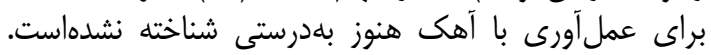

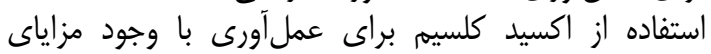

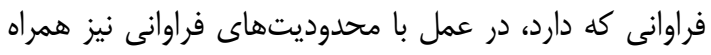

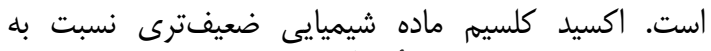
هيدروكسيد سديم بوده و تأثير آن بر ديواره سلونيد الولى كمتر است

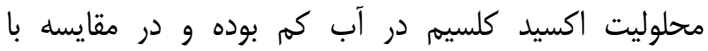

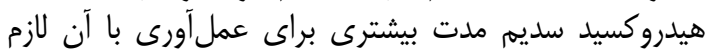

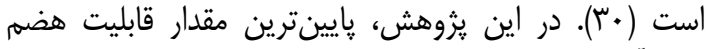

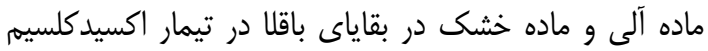

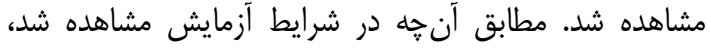

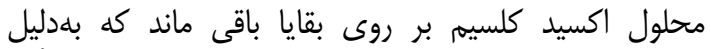

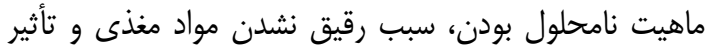

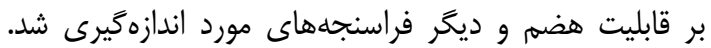

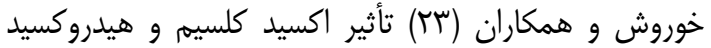

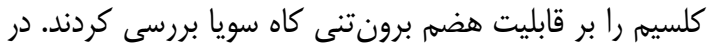

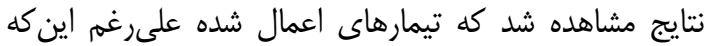
ديواره سلولى كاه سويا را كاهش دادند ادند، اما تأثيرى بر قابلى إليت هضم برونتنى آن نداشتند.

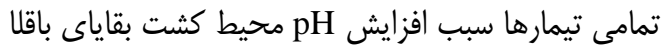

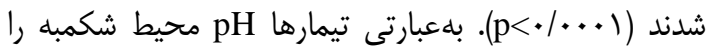

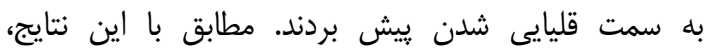

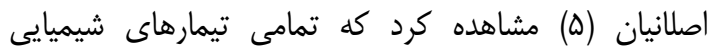

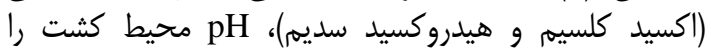

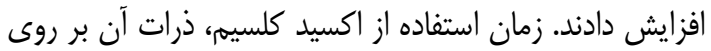

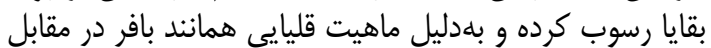

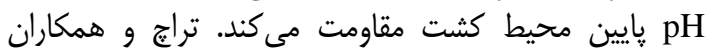

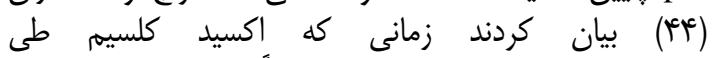

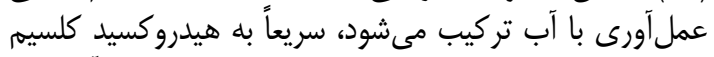

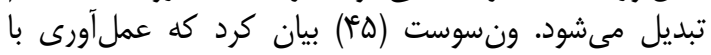
هيدروكسيد سديم باعث تركيب سديم با كربنهاي دئ ديواره كاه كاه 
معنى دار نبود. در يزوهش سلطانى و همكاران (عَّ)، تيمارهاى

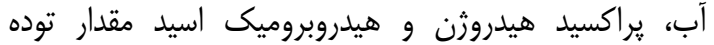
ميكروبى توليد شده و بازده آن را افزيد هيد ايش دادند

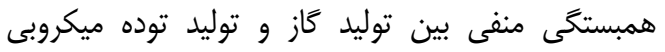

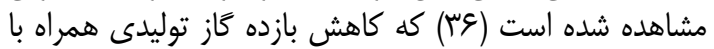

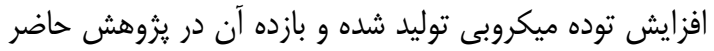

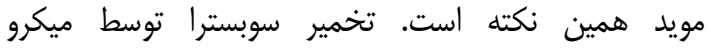

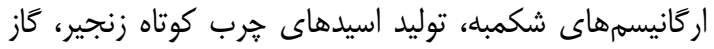

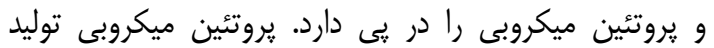

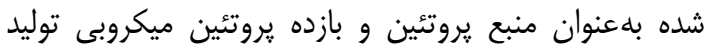

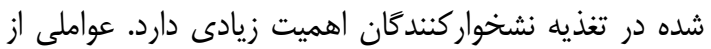

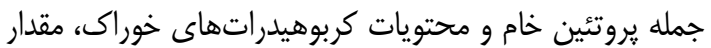

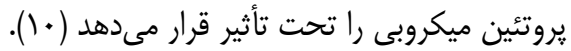

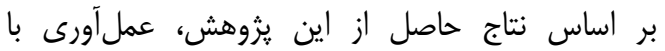

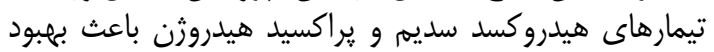

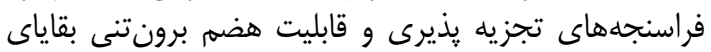

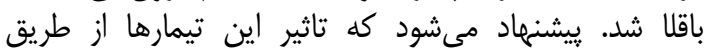
آزمايش هاى عملكردى نيز مورد بررسى قرار كيرد تيرد
محلول كاهش يافته، در نتيجه هضميذيرى، تخمير و توليد

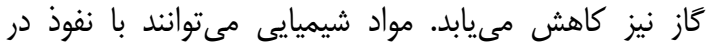

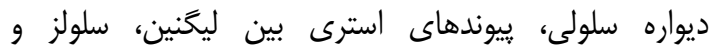

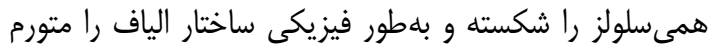

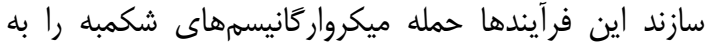

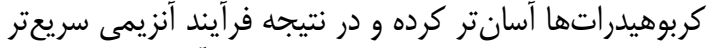

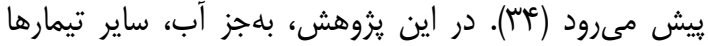

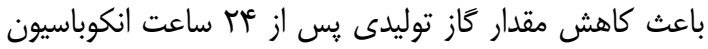
در بقاياى باقلا شدند.

مقدار تودهى ميكروبى توليد شده در تيمار شاهد

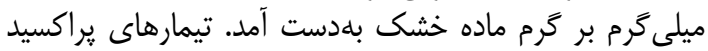

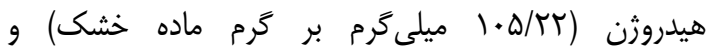

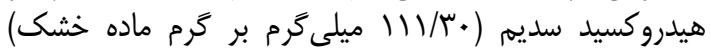

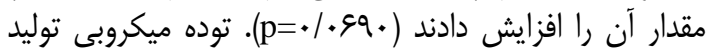

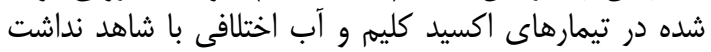

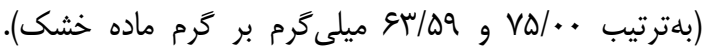

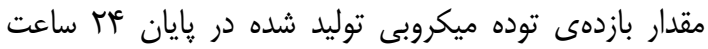

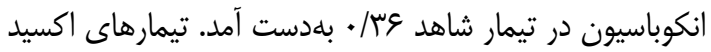

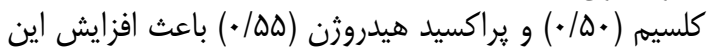

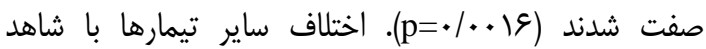

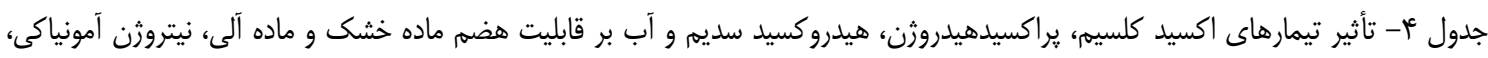
pH

Table 4. Effect of calcium oxide, hydrogen peroxide, sodium hydroxide and water treatments on dry matter and organic matter digestibility, ammoniacal nitrogen, $\mathrm{pH}$ and estimated parameters of vicia faba residues

\begin{tabular}{|c|c|c|c|c|c|c|c|c|}
\hline شازده توده ميايكروبى توليد باعت & 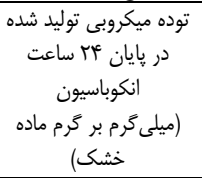 & 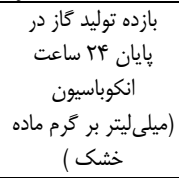 & عامل تفكيك ميلى & $\begin{array}{l}\text { pH } \\
\text { كحتط }\end{array}$ & 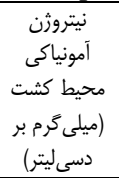 & آلى (درمد مادم & خشم ماده & تيمارها \\
\hline . & $V N / K^{\mathrm{b}}$ & $r \mp V / M^{b}$ & $r / \kappa^{e c d}$ & $g / V r^{c}$ & $r / 9^{b}$ & $\mathrm{~F} / / . \mathrm{b}^{\mathrm{b}}$ & a) $/ . .^{b}$ & شاهد \\
\hline$\cdot 10 \cdot{ }^{a}$ & $V Q / . . b$ & $198 / 99^{d}$ & $\kappa / \mathbb{E}^{\mathrm{ab}}$ & $s / \Lambda e^{a}$ & $r / I V^{d}$ & ५q/..c & $f \cdot 1 . c^{c}$ & اكسيدكلسيم \\
\hline$\cdot / \Delta \Delta^{\mathrm{a}}$ & $1 \cdot \Delta / r r^{a}$ & ل & $r / q v^{a}$ & $\varepsilon / \wedge \Delta^{\mathrm{a}}$ & $r / \Lambda r^{a}$ & $\mathrm{fV} / \ldots \mathrm{b}$ & $\Delta F / \ldots{ }^{\mathrm{a}}$ & يراكسيدهيدروزن \\
\hline$\cdot / \pi 9^{c}$ & $q \mu / \Delta q^{b}$ & $r \wedge \mid / .{ }^{a}$ & $r / 1 l^{d}$ & $s / \mathrm{V}^{\mathrm{b}}$ & $r / \mathrm{r} \Lambda^{\mathrm{cb}}$ & $\mathrm{FV} / . \mathrm{b}^{\mathrm{b}}$ & $\mathrm{eq} / . . \mathrm{b}$ & آب \\
\hline$\cdot / \mathbb{F}^{\mathrm{ab}}$ & $111 / \mu \cdot{ }^{a}$ & $r 1 . / . \mu \mathrm{c}$ & $r / / r^{\mathrm{cb}}$ & s/Av $\mathrm{v}^{\mathrm{a}}$ & $r / \& \Gamma^{c d}$ & $\Delta V / . .^{a}$ & $\Delta \varepsilon / .^{\mathrm{a}}$ & هيدروكسيدسديم \\
\hline אז./. & $11 / v v$ & $1.1 \cdot v \cdot$ & 每. & $.1 \cdot 1$ &.$/ / V$ & $r / 19$. & $1 / \Delta \cdot 1$ & اشتباه معيار ميانكين \\
\hline.$/ . \cdot 19$ & .1 .99$. & $<\cdot / \ldots+\mid$ &.$/ . .14$ & $<\cdot / \cdots+\mid$ & $\cdot / \ldots r$ & ./. rq &.$/ \ldots 1$ & اختلاف معنىدارى \\
\hline
\end{tabular}


1. Afzalzade, A., H. Ghorbani, M. DaneshMesgaran and A. Khadem. 2010. Soaked straw and alfalfa utilization in dairy cattle ration. Journal of Animal production, 12: 37-50 (In Persian).

2. Alemu, T., P. Chairatanyuth, P. Vijchulata and S. Tudsri. 2006. Production and Utilization of crop residue in three agro ecological zones of Eastern Shoa Zone, Ethiopia. Kasetsart Journal, 40: 643-651.

3. Al-Masri, M.R. 2005. Nutritive value of some agricultural wastes as affected by relatively low gamma irradiation levels and chemical treatments. Animal Feed Science and Technology, 96: 1737-1741.

4. AOAC. 2005. Official Methods of Analysis. Association of Official Analytical Chemists. Washington, DC. USA.

5. Aslanian, A. 2014. Effects of processing with gamma ray, calcium oxide and sodium hydroxide on nutritional value of soybean straw. MSc Thesis. Gonbad Kavous Uiversity. 115 pp (In Persian).

6. Aslanian, A., F. Ghanbari, J. Bayat Kouhsar and B. Karimi Shahraki. 2015. Effects of processing with gamma ray, sodium hydroxide and calcium oxide on gas production parameters and digestibility of soybean straw. Journal of Animal Production, 2: 235-248 (In Persian).

7. Babayi, M., F. Ghanbari, A. M. Gharehbash and J. Bayat Kouhsar. 2016. Effects of processing with electron beam, hydrogen peroxide and hydrobromic acid on the nutritional value of vetch wastes. Iranian Journal of Animal Science Research, 8: 441-454 (In Persian).

8. Barlianti, V., D. Dahnum, H. Hendarsyah and H. Abimanyu. 2015. Effect of alkaline pretreatment on properties of lignocellulosic oil palm waste. Procedia Chemistry, 16: 195-201.

9. Basiri, Sh. 2007. Processing and enrichment of fruit and vegetables wastes.Islamic Azad University, Shabestar branch, 159 pp (In Persian).

10. Blummel, M. and E.R. Orskov. 1993. Composition of in vitro gas production and nylon bag degradability of roughages in predicting food intake in cattle. Animal Feed Science and Technology, 40: $109-119$

11. Blummel, M., H. Steingass and K. Becker. 1997. The relationship between gas production, microbial biomass yield and $15 \mathrm{~N}$ incorporation and its implications for the prediction of voluntary feed intake of roughages. British Journal of Nutrition, 77: 911-921.

12. Broderick, G.A. and J.H. Kang. 1980. Automated simultaneous determination of ammonia and total amino acids in ruminal fluid and in vitro media. Journal of Animal Science, 63: 64-75.

13. Chang, V.S. and M.T. Holtzapple. 2000. Fundamental factors affecting biomass enzymatic reactivity. Applied Biochemistry and Biotechnology- part A. Enzyme Engineering and Biotechnology, 86: 5-37.

14. Chaudhry, A.S. 1998. Nutrient composition, digestion and rumen fermentation in sheep of wheat straw treated with calcium oxide, sodium hydroxide and alkaline hydrogen peroxide. Animal Feed Science and Technology, 74: 315-328.

15. Chaudhry, A.S. 2000. Rumen degradation in sacco in sheep of wheat straw treated with calcium oxide, sodium hydroxide and sodium hydroxide plus hydrogen peroxide Animal Feed Science and. Technology, 83: 313-323.

16. Cone, J.W., W. Cline Theil, A. Malestein and A.T. VantKlooster. 1989. Degradation of starch by incubation with rumen fluid. A comparison of different starch sources. Journal of the Science of Food and Agriculture, 49: 173-183.

17. Danesh Mesgaran, M., M. Malakkhahi, B. Heravi Moussavi, A.R. Vakili and A. Tahmasbi. 2010. In situ ruminal degradation and in vitro gas production of chemically treated sesame stover. Journal of Animal and Veterinary Advances, 9: 2256-2260.

18. Ghiasvand, M., K. Rezayazdi and M. Dehghan Banadaki. 2011. The effects of different processing methods on chemical composition and ruminal degradability of canola straw and its effect on fattening performance of male Holstein calves. Journal of Animal Science Researches (Agricultural Science), 22: 93-104 (In Persian).

19. Ghorbani, H. 2007. Study of replacing part of alfalfa hay with straw treated by soaking in dairy cattle ration. MSc Thesis. University of Tehran (In Persian).

20. Harmsen, P., W. Huijgen, L. Bermudez and R. Bakker. 2010. A review of physical and chemical pretreatment processes for lignocellulosic biomass. Food and Biobased Research, 1184: 1-54.

21. Hidenori, A.B.E. and Y. Masaaki. 2000. Effects of ammonation on dry matter digestibility of grasses and legums. Journal of Animal Science, 9: 7-21.

22. Izdorczyk, M.S., L.J. Macri and A.W. MacGregor. 1998. Structural and physicochemical properties of barley non-starch polysaccharides. Alkali extractable $\beta$-glucans and arabinoxylans. Carbohydrate Polymers, 35: 259-269.

23. Khorvash, M., S. Kargar, T. Yalchi and G.R. Ghorbani. 2010. Effect of calcium oxide and calcium hydroxide on the chemical composition and in vitro digestibility of soybean straw. Journal of Food Agricalture and Environment, 8: 356-359.

24. Makkar H.P.S. 2010. "In vitro screening of feed resources for efficiency of microbial protein synthesis" in "In vitro screening of plant resources for extra-nutritional attributes in ruminants: nuclear and related methodologies". Springer, Dordrecht, 107-144. 
25. Makkar, H.P.S., M. Blummel and K. Becker. 1995. Formation of complexes between polyvinyl pyrrolidones or polyethylene glysols and tannins, and their implication in gas production and true digestibility in in vitro techniques. British Journal of Nutrition, 73: 897-913.

26. Mehrez, A.Z. and E.R. Orskov. 1997. A study of the artificial bag technique for determining the digestibility of feed in the rumen. Journal of Agricaltural Science, 88: 645-650.

27. Mosir, N., C. Wyman, B. Dale, R. Elander, Y.Y. lee, M. Holtzapple and M. ladisch. 2005. Feature of promising technologies for pretreatement of lignocellulosic biomass. Bioresoure Technology, 96: 673686.

28. Nigus, A., M. Yusuf and J. Animut. 2014. Subtitution effect of faba bean (Vicia Faba L.) hull to wheat bran on body weight change and carcass characteristics of Afar sheep fed hay as basal diet. Agricultural Science, Engineering and Technology, 2: 1-11.

29. Orskov, E.R. and I. McDonald. 1979. The estimation of protein degradability in the rumen from incubation measurements weighted according to rate of passage. Journal of Agriculture Science, 92: 499-503.

30. Owen, E., T. Klopfenstan and N.A. Urio. 1984. Treatement with other chemicals in straw and other fibrous by-product as feed. Sundstol and Owened. Elsevier Science publishers, Amesterdam, 248273.

31. Pasandi, M. 2014. Determination of digestibility and utilization of ensiled broad bean stover on performance of fattening lambs. Animal Science Journal (Pajouhesh and Sazandegi), 17-24 (In Persian).

32. Sarnklong, C., J.W. Cone, W. Pellikaan and W.H. Hendriks. 2010. Utilization of rice straw and different treatments to improve its feed value for ruminants: A Review. Asian- Australian. Journal of Animal Science, 23: 680-692.

33. SAS. 2003. SAS User's Guide: Statistics, Version 9.1 Edition. SAS Institute, Cary, NC, USA.

34. Selim, A.S.M., J. Pan, T. Takano, T. Suzuki, S. Koike, Y. Kobayashi and K. Tanaka. 2004. Effect of ammonia treatment on physical strength of rice straw, distribution of straw particles and particle associated bacteria in sheep rumen. Animal Feed Science and Technology, 115: 117-128.

35. Sherasia, P.L., M.R. Garg and B.M. Bhanderi. 2017. Pulses and their by-products in animal feed. Food and Agriculture Organization of the United Nations (FAO), $222 \mathrm{pp}$.

36. SoltaniNaseri, K., F. Ghanbari, J. Bayatkouhsar and F. Taliey. 2018. Effect of chemical and biological processing methods on chemical composition, gas production parameters and in vitro digestibility of cicer Arietinum wastes. Research on Animal production, 9: 72-82.

37. Sommart, K., D.S. Parker, P. Rowlinson and M. Wanapat. 2000. Fermentation characteristics and microbial protein synthesis in an in vitro system using cassava, rice straw and dried ruzi grass as substrates. Asian-Australasian Journal of Animal Sciences, 13: 1084-1093.

38. Sun, R.C., J. Tomkinson, Y.X. Wang and B. Xiao. 2000. Physico- chemical and structural characterization of hemicellulose from wheat straw by alkaline proxideextraction. Polymer, 41: 47-57.

39. Sundstut, F. 1988. Straw and other fibrous by-products as feed. Livestock Production Science, 19: 137-157.

40. TavasoliNia, A.R., R. Valizadeh, R. Vakili and S. Sobhani Rad. 2008. Effect of canola straw processed with different levels of sodium hydroxide and urea on performance of Kordish male lambs. 3th Iranian Animal Science Congress, 1-4 (In Persian).

41. Teymouri Chamebon, A., A. Teymori Yanesari, Y. Chashnidel and A. Gafary Sayadi. 2017. Study of chemical composition, quality and ruminal degradability parameters of silaged orange pulp with wheat straw and urea. Research on Animal production, 8: 84-95.

42. Theodorou, M. K., B. A. Williams, M. S. Dhanoa, A. B. McAllan and J. France. 1994. A simple gas production method using a pressure transducer to determine the fermentation kinetics of ruminant feeds. Animal Feed Science and Technology, 48: 185-97.

43. Trach, N. X. 2000. Treatment and supplementation of rice straw for ruminant feeding in Vietnam. Doctor Scientarium Thesis. Agricultural University of Norway. As, Norway.174 pp.

44. Trach, N.X., M. Mo and C. Xuan Dan. 2001. Effects of treatment of rice straw with lime and urea on its chemical composition, gas production and in sacco degradation characteristics. Livestock Research for Rural Development, 13: 117-134.

45. Van Soest, P.J. 1994. Nutritional Ecology of the Ruminant. Cornel University Press, Ithaca, New York, $374 \mathrm{pp}$.

46. Van Soest, P.J. 1982. Nutritional ecology of the ruminant. O\&B Books, Corvallis, OR.

47. Yalchi, T., Sh. Kargar, M. Khorvash and G.R. Ghorbani. 2012. Effect of sodium hydroxide on chemical composition and in vitro digestibility of soybean straw. 3th Iranian Animal Science Congress, 1069-1073. 


\title{
Evaluation of Nutritional Value of Vicia Faba Residues Processed With Some Chemical Compounds Using In Vitro and Nylon Bag Techniques
}

\author{
Azin Alaei ${ }^{1}$, Farzad Ghanbari ${ }^{2}$, Javad Bayat Kouhsar ${ }^{3}$ and Fariba Farivar ${ }^{3}$
}

\author{
1 and 3- Graduated M.Sc. Student and Assistant Professor, Department of Animal Science, Faculty of Agriculture \\ and Natural Resources, Gonbad Kavous University \\ 2- Assistant Professor, Department of Animal Science, Faculty of Agriculture and Natural Resources, Gonbad \\ Kavous University (Corresponding author: farzadghanbari@ yahoo.com) \\ Received: July 25, 2019 \\ Accepted: October 14, 2019
}

\begin{abstract}
This research was conducted to evaluate the nutritional value of vicia faba residues processed with some chemical compounds using in vitro and nylon bag techniques. Treatments included unprocessed vicia faba residues (control) and processed with water (2.5 lit $/ \mathrm{kg} \mathrm{DM}$ ), calcium oxide $(160 \mathrm{~g} / \mathrm{kg} \mathrm{DM})$, hydrogen peroxide $(57 \mathrm{ml} / \mathrm{kg} \mathrm{DM})$ and sodium hydroxide $(50 \mathrm{~g} / \mathrm{kg} \mathrm{DM})$. The chemical compositions of the samples were identified using the standard methods. Ruminal degradability trial was performed using the nylon bag technique. In vitro digestibility of samples was determined by the batch culture method. Processing was effective on the chemical composition of vicia faba residues. Except for water, the other treatments increased Ash and decreased organic matter $(p=0.0001)$. Calcium oxide had the highest effect on the mentioned traits. Crude protein amount was decreased in all treatments $(p=0.0001)$. The lowest amount was observed in calcium oxide and sodium hydroxide treatments. The amount of acid detergent fiber in calcium oxide and hydrogen peroxide treatments was higher than other treatments $(p=0.0017)$. Except for calcium oxide and water, the other treatments increased effective rumen degradability of vicia faba residues at rumen outflow rates of 2,5 and 8 percent/hour $(p<0.0001)$. The hydrogen peroxide had the greatest effect on increase of effective rumen degradability. Sodium hydroxide treatment increased dry matter $(p=0.0001)$ and organic matter $(p=0.0029)$ digestibility. Also, calcium oxide and hydrogen peroxide increased the efficiency of microbial yield $(p=0.0016)$. Totally, the results of this research showed that the sodium hydroxide and hydrogen peroxide treatments had the greatest effect on improving the nutritional value of vicia faba residues.
\end{abstract}

Keywords: Processing, Chemical Compounds, Digestibility, Ruminal Degradability, Vicia Faba Residues 